Vol 11, Issue 11, 2018

\title{
EVALUATION OF USE OF ANALGESICS IN PAIN MANAGEMENT AMONG SURGEONS IN A TERTIARY CARE HOSPITAL
}

\author{
JAYSHREE DAWANE*, KALYANI KHADE, YAMINI INGALE, VIJAYA PANDIT \\ Department of Pharmacology, Bharati Vidyapeeth Deemed University Medical College, Pune, Maharashtra, India. Email: jayshreedawane@ \\ gmail.com \\ Received: 24 May 2018, Revised and Accepted: 29 June 2018
}

\begin{abstract}
Objective: The objective of this study is to evaluate pain and to assess if analgesic prescriptions are according to the World Health Organization guidelines.

Methods: The study was conducted in the Department of Surgery in a tertiary care hospital. Patients with age >18 years, of either sex, admitted to surgery ward were included in the study. Pain assessment was done using a visual analog scale and McGill questionnaire. Information obtained from case paper sheets was recorded, such as name of analgesics, the generic name of prescribed analgesics, dosage, route of administration, frequency, number of analgesics per prescription, and non-pharmacological techniques. Data generated from the questionnaire were entered into an Excel sheet, and percentages were calculated.
\end{abstract}

Results: A total of eight different analgesics were prescribed in the study group. Paracetamol was the maximally prescribed drug (40\%). In $48 \%$ of cases, antacids were given along with analgesics. A majority of analgesics were prescribed in generic names (52\%). No drug was prescribed to almost $18 \%$ cases even though the pain intensity was of mild-to-moderate intensity.

Conclusion: Commonly prescribed drugs were paracetamol + tramadol. Prescription pattern of analgesics is partially deviating from standard guidelines. Generic names were written in the majority of prescriptions, which is in accordance with standard prescription writing.

Keywords: Pain, Analgesics, Observational study.

(C) 2018 The Authors. Published by Innovare Academic Sciences Pvt Ltd. This is an open access article under the CC BY license (http://creativecommons. org/licenses/by/4. 0/) DOI: http://dx.doi.org/10.22159/ajpcr.2018.v11i11.27489

\section{INTRODUCTION}

Pain is one of the most common symptoms for which patients look for medical attention and is also important in making the correct diagnosis. The International Association for the Study of Pain defines pain as "an unpleasant sensory and emotional experience associated with actual or potential tissue damage or described in terms of such damage" [1]. Pain is always a subjective experience and a sensation perceived by the patient. Uncontrolled pain gives a constant feeling of discomfort to the patient, which affecting his/her psychological and functional wellbeing [2].

Patients admitted to the surgery ward suffer from diverse pain, which is managed medically or surgically. In patients' undergone surgical procedure, the purpose of post-operative pain (POP) control is to maintain functional abilities, as well as physical and psychological wellbeing of the patient. This is achieved by providing safe and effective control of acute pain immediately after the intervention. Pain in recently operated patients refrains them from activity leading to weakness, delayed rehabilitation, and ambulation which may further lead to increased risk of deep vein thrombosis [3]. Adequate control of pain is essential to enhance their quality of life, ultimately proceeding to faster recovery [4].

In the year 1996, the American Pain Society introduced the phrase "pain as the $5^{\text {th }}$ vital sign" [5]. This concept was introduced to create an awareness of pain treatment among healthcare professionals. This initiative emphasizes that pain assessment is as important as an assessment of the standard four vital signs - pulse, blood pressure, temperature, and respiration, and every patient should be assessed for pain every time these vital signs are measured. Pain assessment is the first and important step of pain management. There are various standard scales used for pain assessment of which few are patient's scales and few are observer scale. Timely assessment will help us to determine the change in pain intensity score as a response to treatment and to decide if the given analgesic is effective enough or not. One of the objectives of the National Drug Policy is to promote the rational use of drugs by prescribers, dispensers, and consumers. In developing countries, inappropriate analgesic prescribing has been identified among healthcare facilities.

Therefore, this study is planned to obtain the data on pain management and type and appropriateness of analgesics prescribed in a tertiary care hospital.

\section{METHODS}

The study was approved by the Institutional Ethics Committee of Bharati Vidyapeeth Medical College, Pune, Maharashtra (DCGI Reg. No. ECR 518 - BVDU/MC/E51). This trial has been registered with the Clinical Trial Registry of India (Registration No. CTRI/2018/04/013499).

\section{Study site}

The study was conducted in the Department of Surgery in a tertiary care hospital.

\section{Study design}

Prospective, observational, cross-sectional, unicentric, and descriptive type of study.

\section{Study period}

Two months, from March 2017 to May 2017.

\section{Study sample}

Patients with age $>18$ years, of either sex, admitted to surgery ward during the same period and willing to participate in the study. 
Written consent was taken after giving complete information of study. For assessment of pain, a data collection form was designed and validated. Data and information were collected from patients and from case papers within $24 \mathrm{~h}$ of admission

\section{Pain assessment}

There are various standard scales used for pain assessment of which few are patient's scales and few are observer scale. We used visual analog scale (VAS) and McGill Questionnaire for the pain assessment.

\section{VAS}

The VAS is a one-dimensional measure of pain intensity. It is generally regarded as a valid and reliable tool for pain assessment and is the most widely used scale for the pain assessment [6]. It is usually $10 \mathrm{~cm}$ in length and has a marker that the patient moves to the point, indicating his/her pain of intensity. For pain intensity, the scale is anchored by "no pain" at 0 and "worst imaginable pain" at 10 . The following cut points on the pain VAS have been recommended: no pain $(0-0.4 \mathrm{~cm})$, mild pain $(0.5-$ $4.4 \mathrm{~cm})$, moderate pain $(4.5-7.4 \mathrm{~cm})$, and severe pain $(7.5-10 \mathrm{~cm})$ [7].

\section{McGill pain questionnaire (MPQ)}

MPQ is a multi-dimensional pain questionnaire which is designed to measure the sensory, affective, and evaluative aspects of pain and pain intensity. VAS provides data on the intensity of pain, but it does not give any data on the qualities of pain. MPQ is a valid and reliable tool that evaluates both the quality and quantity of pain through the use of unique pain descriptors using a questionnaire which is easy to understand by patients [8].

This questionnaire has the following three sections broadly:

1. What does your pain feel like?

2. How strong is your pain?

3. How does your pain change with time?

The first section with heading as "What does your pain feel like?" describes quality or characteristic of pain. The second section asking "How strong is your pain?" describes the present pain intensity and overall pain experience. The third section with heading as "How does your pain change with time?" is useful in assessing the pattern of pain. The total pain rating index score is obtained by summing the scores obtained from different subscales. First part includes anatomical location of pain and recording quality of pain using different descriptors. Descriptor 1-11 represents sensory dimension of pain, and descriptor 12-15 represent affective dimension of pain. Second part includes recording of present pain intensity using visual analog scale, and assessment of overall pain experience. Last part includes recording of pattern of pain. All these scores are added together to get total pain rating index. As for the MPQ there are no established critical cut points, a higher score indicates worse pain. It can be used to monitor the pain over time and to determine the effectiveness of any intervention. Timely assessment will help us to determine the change in pain intensity score as a response to treatment and to decide if the given analgesic is effective enough or not. No training is required to score and interpret both VAS and MPQ.

Information obtained from case paper sheets which mainly includes details such as name of analgesics as written by the surgeon, generic name of prescribed analgesics (opioid and non-opioid), dosage, route of administration, frequency and duration of administration, number of analgesics per prescription, total number of drugs prescribed, nonpharmacological techniques if advised, and multimodal anesthesia if prescribed was noted down. Patients were followed up on day 7 and 15. If patients were discharged of hospital, follow up was done on telephone. Similar details were noted on follow-up visits.

\section{Statistical analysis}

Data generated from the questionnaire were coded, entered, and statistical analysis was done using Microsoft Excel 2010. Results were expressed in percentage. Frequency distribution tables and chart were prepared from the analysis.

\section{RESULTS}

In this study, the male-to-female ratio was almost similar. About $48 \%$ of patients were male and $52 \%$ of patients were female. The study included patients ranging from 20 years to 80 years of age. Maximum patients were from age group 20-40 years. The mean age was 43 years. Table 1 shows disease-wise distribution of patients. Many patients were admitted for the pain arising out of cancer. Next to it were patients with renal calculi and so on

Fig. 1 shows overall analysis of all the prescriptions. Among them, non-steroidal anti-inflammatory drugs (NSAIDs) were preferred over opioid analgesics. In single-drug prescriptions, paracetamol was the maximum prescribed analgesic (85\%) followed by diclofenac (27\%) and ibuprofen $(4 \%)$. About $11 \%$ prescriptions contained opioid analgesic, i.e., tramadol. To $8 \%$ of cases, no analgesic was prescribed.

Overall, opioid analgesics were prescribed only in $27 \%$ cases (Fig. 2). Fig. 3 shows a graphical representation of combinations of analgesics in prescriptions. Among combinations, paracetamol was used most commonly. Maximum combinations (72\%) were of paracetamol + diclofenac. Followed to it was paracetamol + ibuprofen (12\%), paracetamol + tramadol $(14 \%)$, and least used combination was diclofenac + tramadol $(2 \%)$

Fig. 4 shows the distribution of drugs according to the branded and generic name. Paracetamol was the only prescribed with the generic name in 83 cases, whereas all remaining drugs such as diclofenac (13), tramadol (7), ibuprofen (0), and other drugs $(0)$ were prescribed with the brand name.

Fig. 5 shows that $74 \%$ of cases received acid-reducing drugs along with analgesics. Of these $74 \%$ cases, who were receiving acid-reducing drugs as an adjuvant, were majorly belonging to NSAIDs class. Proton pump inhibitors and $\mathrm{H} 2$ blockers were the two classes of drugs used for the purpose of inhibition of acid secretion. Among them, rantac (ranitidine) and Pan-D (pantoprazole-domperidone) were prescribed maximally.

\section{DISCUSSION}

Pain is the warning signal of the underlying disease. When it is unbearable and interferes with daily activities, it becomes a compelling reason for

Table 1: Percentage distribution of illness

\begin{tabular}{ll}
\hline Diseases & Percentage \\
\hline Cancer & 30 \\
Renal calculi & 17 \\
Fracture & 13 \\
Appendicitis & 5 \\
Hernia & 8 \\
Pancreatitis & 3 \\
Cholelithiasis & 4 \\
Abscess/cellulitis & 8 \\
GIT (intestinal obstruction, & 5 \\
intussusception, intestinal perforations) & \\
Others & 7 \\
\hline
\end{tabular}

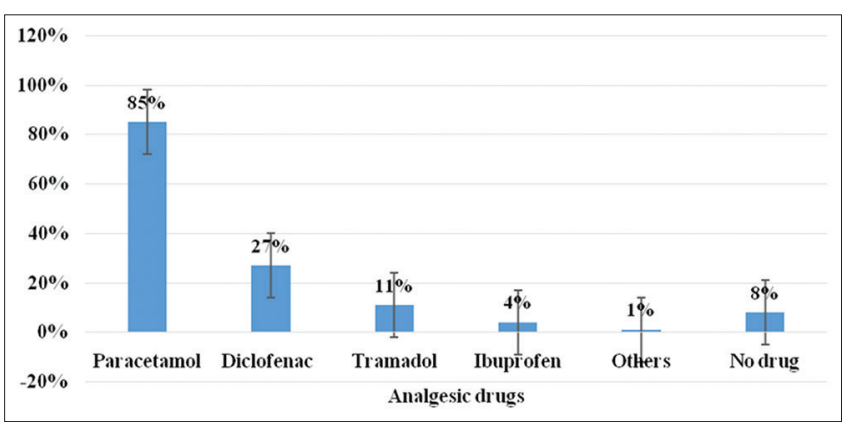

Fig. 1: Percentages of analgesics prescribed as a single drug 


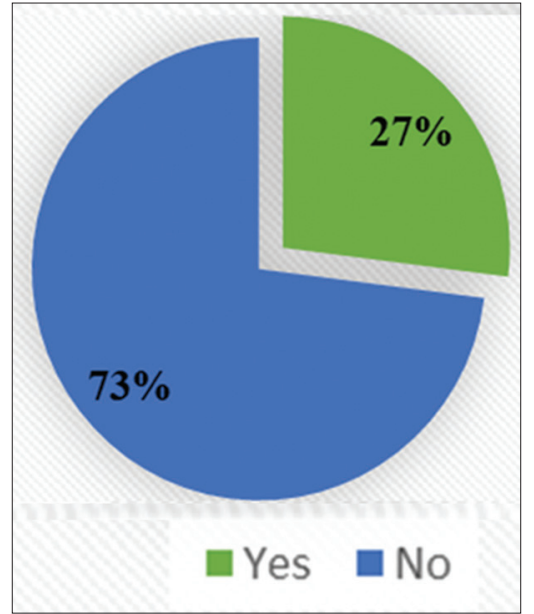

Fig. 2: Percentage of cases received opioid analgesic

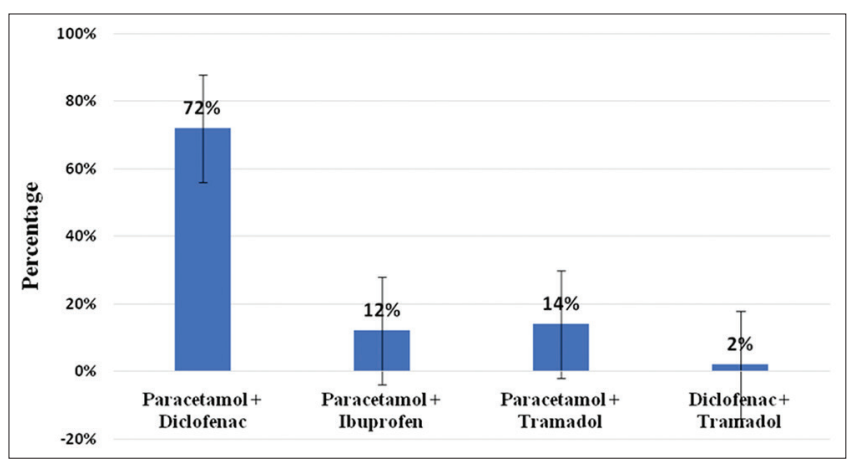

Fig. 3: Percentage of drug used in combinations

seeking medical attention [9]. Surgery wards also have patients operated for various ailments. It is essential to control POP in such patients to maintain their functional abilities as well as physical and psychological well-being [10]. Uncontrolled pain can have several physical and psychological ill effects and if left untreated can further affect almost all body systems. Pain in recently operated patients refrains them from activity leading to weakness, delayed rehabilitation, and ambulation which may further lead to increased risk of deep vein thrombosis [11]. Adequate control of pain in such patients can enhance their quality of life, ultimately proceeding to faster recovery [3] this will further help in reducing time spent in the hospital, readmissions for further pain management and development of chronic pain. One of the objectives of the National Drug Policy is to promote the rational use of drugs by prescribers, dispensers, and consumers. In developing countries, inappropriate analgesic prescribing has been identified among healthcare facilities. Undertreatment of pain will let the patient suffer from pain and with overtreatment patient will unnecessary be exposed to the adverse effects of drugs. Therefore, this one-time study was planned to obtain the data on pain management and type and appropriateness of analgesics prescribed in a tertiary care hospital. The study of prescribing pattern is a significant constituent of a medical audit which helps in monitoring, evaluating, and making required modifications in the prescribing practices to attain a rational medical care. The prescription audit is an important part of drug utilization studies. Inappropriate use of analgesics may either cause inadequate pain relief or increased incidence of adverse effects, habituation, abuse potential, and the emergence of tolerance requiring an increased amount of drugs. Both the situations, inadequate control and unnecessary prescription of analgesics can have a negative influence on the health of the patient, and hence, it is utmost important to give adequate analgesia with appropriate analgesics.

Table 1 includes patients suffering from the different ailments, such as renal calculi, appendicitis, and fractures, and admitted in surgery ward

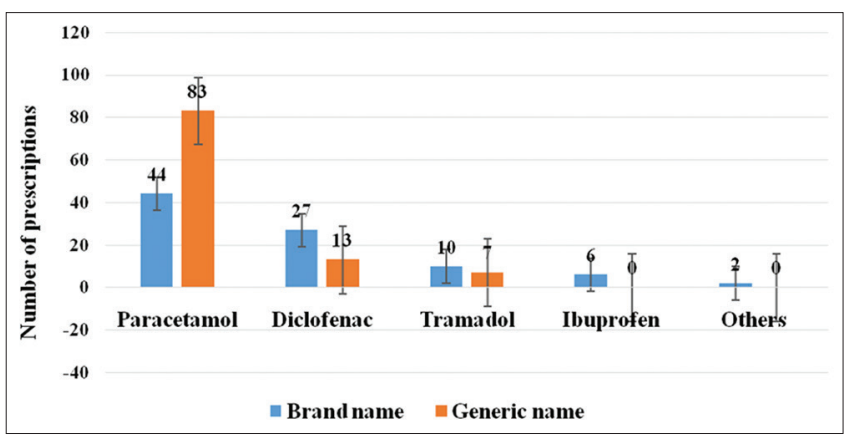

Fig. 4: Distribution of drug used as branded and generic

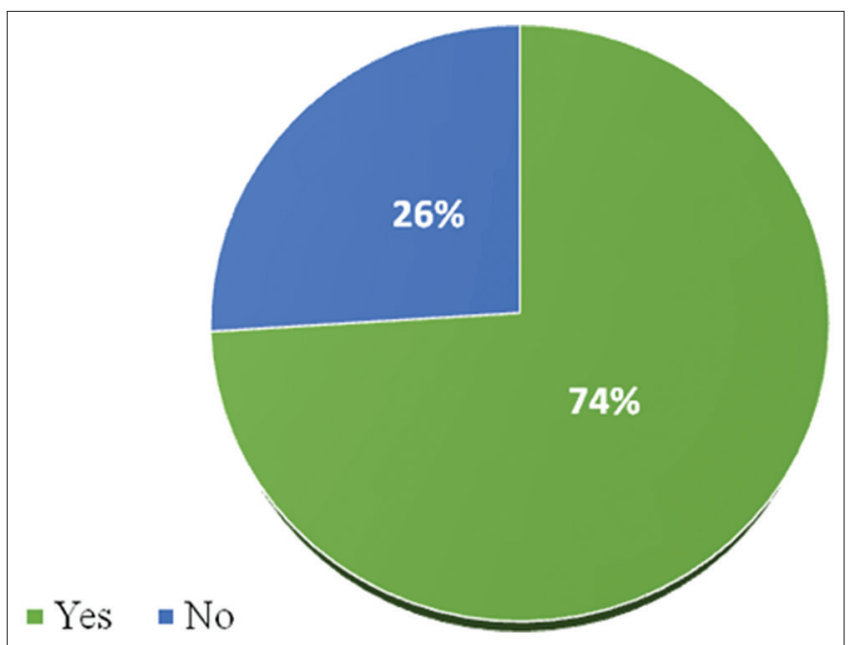

Fig. 5: Percentages of cases given acid-reducing drug

for the treatment were included in the study. The highest number of patients was from 20 to 40 years age group. Lowest number of patients was from above 80 age group. Mean age of patients was 43 years. The male-to-female ratio in the study was almost similar.

Paracetamol, diclofenac, ibuprofen, and tramadol were the most commonly used analgesics in the present study. Majority of them belong to NSAIDs group. In the maximum number of cases, paracetamol was given by injectable route. Furthermore, in case of combinations of an oral and a parenteral analgesic, parenteral analgesic was given as the starting dose and then oral analgesic as the maintenance dose. Mohammed et al. stated that paracetamol was the most commonly prescribed NSAID, which is similar to the findings that we have obtained [12]. Similar results are seen from a prescription study which was done in Medicine department in Dhaka, Bangladesh, by Rahman et al., where paracetamol was the most common prescribed followed by diclofenac [13]. However, from some studies, different observations are seen as, in a study done by Paul et al. [14] and Seager et al. [15], Ibuprofen was the most common analgesic prescribed, may be owing to its less gastric adverse effects against other NSAIDs [16]. This deviates from the results obtained from our study. The analgesics prescribed were found to be appropriate for the indications. However, in few conditions, it was mild analgesic for moderate pain. The duration for which the analgesics were prescribed was also found to be appropriate. Opioid analgesic was found to be prescribed in only $11 \%$ of cases.

The choice of analgesics depends on the severity of pain. According to the severity for mild-to-moderate pain, single analgesics are sufficient, but for moderate-to-severe pain two or more than two analgesics are required. This is also dependent on the efficacy and potency of the analgesic.

There were 12 cases even though complained of pain did not receive any analgesic drug. Nearly $50 \%$ ( 6 cases) of cases were having the moderate 
intensity of pain, and 33\% (4 cases) were in severe pain. According to the World Health Organization (WHO) analgesic ladder guideline, all those patient should have received NSAID + weak opioid drug, but no analgesic drug was prescribed. This shows the slight deviation in the use of analgesic from the standard guideline.

Combination of analgesics plays an extremely valuable role in the management of pain. Drugs used in combinations were paracetamol with diclofenac, ibuprofen, and even with tramadol. Paracetamol with Ibuprofen shows a synergistic analgesic effect, and this combination is routinely used for the treatment of pain. Overall paracetamol was used most frequently, may be because of adequate analgesic action with additional antipyretic effect and least number of side effects, especially no incidence in causing GI side effects, providing better its safety profile among all NSAIDs that too at very less cost. Opioidacetaminophen combinations are recommended in the WHO analgesic ladder for moderate-to-severe intensity of pain [17]. Tramadol with acetaminophen combination provides the potential benefits by increasing efficacy and reducing the adverse effects. Both the component have a different mechanism of action and the combination shows a synergistic effect in pain relief [18]. The goal of the therapy is to facilitate patient compliance, simplify prescribing and improve efficacy without increasing adverse effects and is achieved with combination therapy [19]. A majority of the analgesics were prescribed correctly as regards the strength, frequency, and duration of use. Prescribing less expensive generic drugs is one method of curtailing prescription drug expenditures. In this study, generic paracetamol was prescribed in 83 (65 \%) while branded paracetamol was prescribed in 44 (35\%). Diclofenac with generic name prescribed in $13(33 \%)$ and branded name in 27 (67\%). Tramadol prescribed mostly with brand name only. Drugs when prescribed with generic name definitely help to reduce the cost of the treatment. In the present study, it is in favor of the same and supporting national drug policy.

Analgesics used in the study were from both the groups NSAIDs and opioids. Paracetamol and diclofenac was the most commonly used drug in this study. NSAIDs are known to produce adverse effects such as gastric irritation, nausea, vomiting, ulceration, bleeding, and occult blood loss in stools. To minimize these side effects, NSAIDs are prescribed with acid-reducing agents. Proton pump inhibitors and $\mathrm{H} 2$ blockers were prescribed in $74 \%$ of cases in the present study. However, according to the American College of Gastroenterology, only the patients at high risk for hemorrhage and perforation with aspirin and other NSAID-induced ulcers should be considered for prophylaxis. The commonly used drugs for this purpose are misoprostol and proton pump inhibitors [20]. Patients with prior history of the gastrointestinal event (ulcer and hemorrhage), age above 60 year those who require high dosages of NSAIDs, those who are taking corticosteroids and anticoagulants concurrently are considered as the high-risk patients who require the gastro-protective agents with NSAIDs. There are various adverse effects of long-term PPI therapy as decreased absorption of calcium, magnesium, Vitamin B 12, etc. this may lead to significant reduction in bone mineral density in elderly causing increased fracture risk [21]. Hence, PPI therapy should be personalized and given based on indications. H2 receptor antagonists have been shown to prevent only duodenal ulcer, and therefore, they have limited value in the prophylaxis of NSAIDs-induced gastric ulcers and hence not recommended [22]. However, in the present study, it was prescribed for prophylaxis which cannot be justified.

The study was performed to analyze the use of analgesics by surgeons in patients of surgery ward. According to the WHO analgesic ladder depending on the intensity and type of pain appropriate analgesics should be prescribed. In the present study, pain was assessed with VAS and MPQ, and the data about drug use was obtained from case sheets. It was encouraging that in the majority of cases analgesics were prescribed in accordance with the WHO guidelines. In very few cases, no analgesic was given even though pain was mild-to-moderate intensity. This should be addressed to reduce the patient's sufferings. Inappropriate use of acid-reducing agents was observed with the analgesics which can be reduced. From the study, it was observed that in most of the cases drug combinations were used which enhanced the efficacy and reduced adverse effects because of synergistic combinations of analgesics. Most of the drugs were prescribed with the generic names which were following the National drug policy.

\section{CONCLUSION}

Our study showed that pain management in the tertiary healthcare center was largely in accordance with the National Drug Policy and Standard treatment guidelines. Commonly prescribed drugs were paracetamol and diclofenac combination. A majority of prescriptions were with generic names which were according to the National drug policy. In very few cases, no analgesic was given even though pain was of mild-to-moderate intensity. This should be addressed to reduce the patient's sufferings. In most of the cases, analgesics were given with acid-reducing agents. Measures are required to prevent the irrational use of acid-reducing agents. In a very few cases, prescription pattern of analgesics was slightly deviating from standard guidelines. Nevertheless, this can be upgraded by conducting training and educational programs.

\section{LIMITATIONS}

Our study was carried out in a single tertiary care hospital in India and does not represent all the surgeons in India. In addition, the outpatients and patients from different departments were not considered. An attempt should be made to carry out such studies with larger geographic coverage, with a bigger sample size, and at multiple centers. This will invariably increase the sample size and will give us a broader picture of the plight of the entire state or the entire country.

\section{FINANCIAL SUPPORT AND SPONSORSHIP}

This study has been approved and received funding from Indian Council of Medical Research (ICMR).

\section{CONFLICTS OF INTEREST}

None.

\section{REFERENCES}

1. Carr DB, Goudas LC. Acute pain. Lancet 1999;353:2051-8.

2. Kamble S, Poul B, Udapurkar P. Bilayer tablet of tramadol and gabapentin for combination pharmacotherapy of neuropathic pain: Development and characterization. Int J App Pharm 2018;10:100-7.

3. Lovich-Sapola J, Smith CE, Brandt CP. Postoperative pain control. Surg Clin North Am 2015;95:301-18.

4. Cervero F, Laird JM. Visceral pain. Lancet 1999;353:2145-8.

5. Booss J, Drake A, Kerns RD, Ryan B, Wasse L. Pain as the $5^{\text {th }}$ Vital Sign [Toolkit on the Internet]. Illinois: Joint Commission on Accreditation of Healthcare Organizations; 2000.

6. Mohammed E, Imad S. Validity of patient-reported outcome measurement information system health assessment questionnaire (PROMIS HAQ) for assessing disease activity in Iraqi patients with active rheumatoid arthritis. Int J Pharm Pharm Sci 2014;332-4.

7. Jensen MP, Chen C, Brugger AM. Interpretation of visual analog scale ratings and change scores: A reanalysis of two clinical trials of postoperative pain. J Pain 2003;4:407-14.

8. Hawker GA, Mian S, Kendzerska T, French M. Measures of adult pain: Visual analog scale for pain (vas pain), numeric rating scale for pain (NRS pain), McGill pain questionnaire (MPQ), short-form McGill pain questionnaire (SF-MPQ), chronic pain grade scale (CPGS), short form-36 bodily pain scale (SF-36BPS), and measure of intermittent and constant osteoarthritis pain (ICOAP). Arthritis Care Res 2011;63:S240-52.

9. Dwicandra NO, Setiyadi AP. Comparison of pain score in osteoarthritis patients treated with a combination of Diacerein and Meloxicam and Meloxicam alone. Int J Pharm Pharm Sci 2017;9:69-73.

10. Katz N. The impact of pain management on quality of life. J Pain Symptom Manage 2002;24:S38-47.

11. Dolin SJ, Cashman JN, Bland JM. Effectiveness of acute postoperative pain management: I. Evidence from published data. Br J Anaesth 
2002;89:409-23.

12. Al-Homrany MA, Irshaid YM. Pharmacoepidemiological study of prescription pattern of analgesics, antipyretics, and nonsteroidal anti-inflammatory drugs at a tertiary health care center. Saudi Med J 2007;28:369-74.

13. Rahman MS, Begum ZA, Samad MK. Prescribing pattern of nonsteroidal anti-inflammatory drugs at outpatient departments of teaching hospitals. Bangladesh J Pharmacol 2007;2:1-6.

14. Paul AD, Chauhan CK. Study of usage pattern of nonsteroidal antiinflammatory drugs (NSAIDs) among different practice categories in Indian clinical setting. Eur J Clin Pharmacol 2005;60:889-92.

15. Seager JM, Cullen DJ, Pearson G, Holmes S, Doherty M, Wilson JV, et al. Ibuprofen versus other nonsteroidal anti-inflammatory drugs: Use in general practice and patient perception. Aliment Pharmacol Ther 2000;14:187-91.

16. Henry D, Lim LL, Rodriguez LA, Gutthann S, Carson JL, Griffin M. Variability in risk of gastrointestinal complications with individual nonsteroidal anti-inflammatory drugs: Results of a collaborative meta- analysis. Br Med J 1996;312:1563-6.

17. Schug SA, Zech D, Dörr U. Cancer pain management according to WHO analgesic guidelines. J Pain Symptom Manage 1990;5:27-32.

18. Pergolizzi JV Jr., van de Laar M, Langford R, Mellinghoff HU, Merchante IM, Nalamachu S, et al. Tramadol/paracetamol fixed-dose combination in the treatment of moderate to severe pain. J Pain Res 2012;5:327-46.

19. Tallarida RJ, Raffa RB. Testing for synergism over a range of fixed ratio drug combinations: Replacing the isobologram. Life Sci 1996;58:PL 23-8

20. Lai KC, Lam SK, Chu KM, Wong BC, Hui WM, Hu WH, et al. Lansoprazole for the prevention of recurrences of ulcer complications from long-term low-dose aspirin use. N Engl J Med 2002;346:2033-8.

21. Rostom A, Dube C, Wells G, Tugwell P, Welch V, Jolicoeur E, et al. Prevention of NSAID-induced gastroduodenal ulcers. Cochrane Database Syst Rev 2002;4:CD002296.

22. Lanza FL. A guideline for the treatment and prevention of NSAIDinduced ulcers. Am J Gastroenterol 1998;93:2037-46. 